\title{
Red meat allergy induced by tick bites: A Norwegian case report
}

\author{
${ }^{1}$ Section of Clinical Allergy, Department of Occupational Diseases and \\ ${ }^{2}$ Section of Gastroenterology, Department of Medicine, Haukeland University Hospital, Bergen, Norway \\ ${ }^{3}$ Department of Clinical Medicine, University of Bergen, Bergen, Norway
}

\section{KEY WORDS}

food allergy; red meat; galactose-alpha1,3-galactose (alpha-gal); tick-bite

\section{Corresponding author}

Gülen Arslan Lied

Section of Clinical Allergy

Department of Occupational Diseases

Haukeland University Hospital

5021 Bergen, Norway

E-mail: Gulen.Arslan@uib.no

Phone: +4793411359

\section{Doi}

10.23822/EurAnnACI.1764-1489.04

\begin{abstract}
Summary
Food allergies, especially delayed hypersensitivity reactions, are often challenging for both patients and clinicians. Here, we report the case of a 64-year-old man who had allergic reactions six hours after eating a meal containing red meat. He reported that he had several tick bites in months before the reaction. High serum specific IgE levels of alpha-gal confirmed the diagnosis of alpha-gal allergic reaction with delayed onset after red meat ingestion caused by tick bite.
\end{abstract}

\section{Introduction}

Food allergy is by definition an immunologically mediated hypersensitivity reaction, which can be immunoglobulin $\mathrm{E}$ (IgE) or non-IgE mediated. Allergic reactions can also be divided into 2 categories according to onset of symptoms, as immediate (within the 2 hours after intake of allergens) or late-phase (delayed) reactions (within 2-24 hours).

The prevalence of specific allergic sensitization may vary depending on the geographical region considered. Allergy to red meat used to be considered unusual, but some cases have been reported in different countries including USA, Australia and several European countries such as Germany, France, Switzerland, Italy and Sweden (1-3).

Here, we report the first case of an allergy to red meat induced by tick bites observed in Norway.

\section{Case report}

A 64-year-old male residing in Bergen, Western coast of Norway, presented with pruritus after eating a meal containing meat. He was healthy before, and had not used any medication. According to atopic background, he had no atopy before, on himself and on his first degree relatives.

Since approximately 4 years ago, he was having sudden rash and itching on the whole body. After several similar episodes, he noticed that symptoms occurred after consumption of red meat, especially beef (ox and cow meat) and sheep meat. He could eat a small amount of bacon without any problem, but not a normal meal size of pork meat. Otherwise, he had no symptoms after consumption of chicken, fish and turkey, and he tolerated other foods including milk. This started during a vacation abroad, after consumption of a quite big size of beef meal. Six 
hours later, an allergic reaction started, with itching and afterwards urticaria on the whole body, especially thigh, chest and abdomen, but not on face. He had no dyspnea, dizziness, nausea or diarrhea. After this, he had several episodes with same symptoms after the intake of red meat. Usually, urticaria lasted 1 or 2 day and nights, and itching disappeared within 16-18 hours if he did not use any antihistamines. As long as he avoided eating red meat, things went smoothly. After detailed history, the patient reported that he had bought an abandoned farm some years ago, and that he was being repeatedly bitten by ticks.

We performed allergological examination at our department. Skin prick test with raw ox and sheep meat were negative, and skin prick test using a standard food allergen panel with six allergens (egg white, cow's milk, codfish, wheat flour, peanut, and soy) were also negative. Blood samples for specific $\operatorname{IgE}$ against ox, lamb, pork and elk were high (sIgE: 40.2; 10.6; 24.9 and 8.2, respectively; and upper normal limit of sIgE for these are $<0,35$ $\mathrm{kU} / \mathrm{L})$. Moreover, the IgE antibodies specific for alpha-1,3-galactose (alpha-gal) were extremely high (sIgE: $>100 \mathrm{kU} / \mathrm{L}$ ), and the positivity of this test gives the explanations of his reactions. Otherwise, routine blood tests and other allergy parameters in serum such as total IgE, specific IgE against standard food allergy panel with six allergens (egg white, cow's milk, codfish, wheat flour, peanut, and soy), tryptase and eosinophilic cationic protein were within the normal reference range. Thus, alpha-gal allergy has been explained to the patient, and continuing the diet without consumption of red meat was recommended.

\section{Discussion}

In our knowledge, this is the first published case to report an allergy to red meat induced by tick bites which has been observed in our country. Even though this is a rare allergic reaction, IgE-mediated allergy to the alpha-gal epitope has been described in few similar cases from other countries in the literature (4), and the prevalence of specific allergic sensitization may vary depending on the geographical region considered (5).

Red meat ingestion can cause a delayed reaction by several hours, because of the delayed release of $\mathrm{IgE}$ alpha-gal during the digestion process. The specific IgE antibodies to alpha-gal are a good marker of allergy to foods such as meat from mammals, and this was discovered for the first time by Commins et al. (6). Alpha-gal allergy is a reaction to Galactose-alpha-1,3-galactose, whereby the body is overloaded with IgE antibodies on contact with the carbohydrate. Bites from the tick, which transfer this carbohydrate to the person's skin, have been implicated in the development of this delayed allergic response which is triggered by the consumption of mammalian red meat products (7). Researchers still do not know which specific component of tick saliva causes the reaction. A typical allergic reaction to alpha-gal has a delayed onset, occurring 3-8 hours after the consumption of mammalian meat products, instead of the typical rapid onset with most food allergies. After the delayed onset, the allergic response is typical of most IgE mediated food allergies, which include symptoms from gastrointestinal tract, severe whole-body itching, angioedema, and possible anaphylaxis.

In the literature, the first report of alpha-gal-specific IgE-mediated allergy was from the cases of systemic anaphylaxis after the infusion of cetuximab, a chimeric mouse-human antibody that is used for cancer therapy, and bears the carbohydrate epitope on the mouse Fab portion (8). Moreover, specific IgE to alpha-gal was demonstrated to underlie some cases of systemic anaphylaxis after eating red meat (beef, lamb and pork, but not chicken or fish), gelatin or pork kidney (9). Initial cases of anaphylaxis to alpha-gal were described in the USA, and epidemiological evidence indicated a close relationship with previous exposure to tick bites $(4,7)$. In Europe, cases of delayed anaphylaxis after red meat intake in relation to IgE-mediated alpha-gal sensitization were subsequently described $(1,2)$.

The diagnosis of meat allergy is based on the presence of clinical signs after ingesting meat. In our case, the link between red meat ingestion and allergic reactions was suspected, and a detailed history was also revealed that he was being bitten repeatedly by tick after he bought an abandoned farm some years ago. The assay for serum specific alpha-gal $\operatorname{IgE}$ antibodies was a very significant contribution to diagnosis and helped to reassure the patient. Our patient continued to avoid consumption of red meat and no further allergic reaction were observed.

\section{Conclusion}

Even though red meat allergy induced by tick bites is unusual, some cases have been described in different countries during the last decades. Allergic reactions occur 3-8 hours after the consumption of mammalian meat products (a delayed onset). High serum specific IgE levels of alpha-gal in patients with clinical suspicion of red meat allergy would have a high positive predictive value on diagnosis. An alpha-gal allergy does not require the afflicted to become a vegetarian since poultry and fish do not trigger such a reaction.

\section{References}

1. Calamari AM, Popa M, Villalta D, Pravettoni V. Alpha-gal anaphylaxis: the first case report in Italy. Eur Ann Allergy Clin Immunol. 2015; 47:161-2.

2. Guillier A, Fauconneau A, De Barruel F, Guez S, Doutre MS. Allergic hypersensitivity to red meat induced by tick bites: A French case report. Eur J Dermatol. 2015;25:277.

3. Wuerdeman MF, Harrison JM. A case of tick-bite-induced red meat allergy. Mil Med. 2014;179:473-5.

4. Kaloga M, Kourouma S, Kouassi YI, Ecra EJ, Gbery IP, Allou AS, Diabate A, Djeha D,Sangaré A, Yoboue YP. Allergy to Red Meat: A Diagnosis Made by the Patient and Confirmed by an Assay for IgE 
Antibodies Specific for Alpha-1,3-Galactose. Case Rep Dermatol. 2016;8:10-3.

5. Plats-Mills TAE, Schuyler AJ, Tripathi A, et al. Anaphylaxis to the carbohydrate side chain alpha-gal. Immunol Allergy Clin North Am. 2015:35:247-60.

6. Commins S, Satinover S, Hosen J, Mozena J, Borish L, Lewis B: Delayed anaphylaxis, angioedema, or urticaria after consumption of red meat in patients with IgE antibodies specific for galactose-alpha-1,3-galactose. J Allergy Clin Immunol. 2009;123:426-33.

7. Gonzalez-Quintela A, Dam Laursen AS, Vidal C, Skaaby T, Gude F, Linneberg A. IgE antibodies to alpha-gal in the general adult population: relationship with tick bites, atopy, and cat ownership. Clinical and Experimental Allergy. 2014;44:1061-8.

8. Chung $\mathrm{CH}$, Mirakhur B, Chan E et al. Cetuximab-induced anaphylaxis and IgE specific for galactose-alpha-1,3-galactose. N Engl J Med. 2008;358:1109-17.

9. Araujo RN, Franco PF, Rodrigues H, Santos LCB, McKay C, Sanhueza CA, Brito N, Azevedo MA, Venuto AN, Cowan PJ, Almeida IC, Finn MG, Marques AF. Amblyomma sculptum tick saliva: $\alpha$-Gal identification, antibody response and possible association with red meat allergy in Brazil. International Journal of Parasitology. 2016;46:213-20. 